\title{
Numerical Analysis for Stochastic Partial Differential Delay Equations with Jumps
}

\author{
Yan $\mathrm{Li}^{1,2}$ and Junhao $\mathrm{Hu}^{3}$ \\ ${ }^{1}$ Department of Control Science and Engineering, Huazhong University of Science and Technology, Wuhan 430074, China \\ ${ }^{2}$ College of Science, Huazhong Agriculture University, Wuhan 430074, China \\ ${ }^{3}$ College of Mathematics and Statistics, South-Central University for Nationalities, Wuhan 430074, China
}

Correspondence should be addressed to Junhao Hu; hujunhao@yahoo.com.cn

Received 3 January 2013; Accepted 21 March 2013

Academic Editor: Xuerong Mao

Copyright (C 2013 Y. Li and J. Hu. This is an open access article distributed under the Creative Commons Attribution License, which permits unrestricted use, distribution, and reproduction in any medium, provided the original work is properly cited.

We investigate the convergence rate of Euler-Maruyama method for a class of stochastic partial differential delay equations driven by both Brownian motion and Poisson point processes. We discretize in space by a Galerkin method and in time by using a stochastic exponential integrator. We generalize some results of Bao et al. (2011) and Jacob et al. (2009) in finite dimensions to a class of stochastic partial differential delay equations with jumps in infinite dimensions.

\section{Introduction}

The theory and application of stochastic differential equations have been widely investigated [1-7]. Liu [2] studied the stability of infinite dimensional stochastic differential equations. For the numerical analysis of stochastic partial differential equations, Gyöngy and Krylov [8] discussed the numerical approximations for linear stochastic partial differential equations in whole space. Jentzen et al. [9] studied the numerical simulations of nonlinear parabolic stochastic partial differential equations with additive noise. Kloeden et al. [10] gave the error analysis for the pathwise approximation of a general semilinear stochastic evolution equations.

By contrast, stochastic partial differential equations with jumps have begun to gain attention [11-15]. Röckner and Zhang [15] considered the existence, uniqueness, and large deviation principles of stochastic evolution equation with jump. In [12], the successive approximation of neutral SPDEs was studied. There are few papers on the convergence rate of numerical solutions for stochastic partial differential equations with jump, although there are some papers on the convergence rate of numerical solutions for stochastic differential equations with jump in finite dimensions [16, 17].

Being motivated by the papers $[16,17]$, we will discuss the convergence rate of Euler-Maruyama scheme for a class of stochastic partial delay equations with jump, where the numerical scheme is based on spatial discretization by Galerkin method and time discretization by using a stochastic exponential integrator. In consequence, we generalize some results of Bao et al. (2011) and Jacob et al. (2009) in finite dimensions to a class of stochastic partial delay equations with jump in infinite dimensions. The rest of this paper is arranged as follows. We give some preliminary results of Euler-Maruyama scheme in Section 2. The convergence rate is discussed in Section 3.

\section{Preliminary Results}

Throughout this paper, let $\left(\Omega, \mathscr{F},\left\{\mathscr{F}_{t}\right\}_{t \geq 0}, \mathbb{P}\right)$ be a complete probability space with some filtration $\left\{\mathscr{F}_{t}\right\}_{t \geq 0}$ satisfying the usual conditions (i.e., it is right continuous and $\mathscr{F}_{0}$ contains all $\mathbb{P}$-null sets). Let $\left(H,\langle\cdot, \cdot\rangle_{H},\|\cdot\|_{H}\right)$ and $\left(K,\langle\cdot, \cdot\rangle_{K},\|\cdot\|_{K}\right)$ be two real separable Hilbert spaces. We denote by $(\mathscr{L}(K, H)$, $\|\cdot\|)$ the family of bounded linear operators. Let $\tau>0$ and $D([-\tau, 0], H)$ denote the family of right-continuous function and left-hand limits $\varphi$ from $[-\tau, 0]$ to $H$ with the norm $\|\varphi\|_{D}=\sup _{-\tau \leq \theta \leq 0}\|\varphi(\theta)\|_{H} . D_{\mathscr{F}_{0}}^{b}([-\tau, 0], H)$ denotes the family of almost surely bounded, $\mathscr{F}_{0}$-measurable, $D([-\tau, 0], H)$ valued random variables. For all $t \geq 0, X_{t}=\{X(t+\theta):-\tau \leq$ $\theta \leq 0\}$ is regarded as $D([-\tau, 0], H)$-valued stochastic process. 
Let $T$ be a positive constant. For given $\tau \geq 0$, consider the following stochastic partial differential delay equations with jumps:

$$
\begin{aligned}
d X(t)= & {[A X(t)+f(X(t), X(t-\tau))] d t } \\
& +g(X(t), X(t-\tau)) d W(t) \\
& +\int_{\mathbb{Z}} h(X(t), X(t-\tau), u) N(d t, d u)
\end{aligned}
$$

on $t \in[0, T]$ with initial datum $X(t)=\xi(t) \epsilon$ $D_{\mathscr{F}_{0}}^{b}([-\tau, 0], H),-\tau \leq t \leq 0$. Here $(A, D(A))$ is a self-adjoint operator on $H$. $\{W(t), t \geq 0\}$ is $K$-valued $\left\{\mathscr{F}_{t}\right\}_{t \geq 0}$-Wiener process defined on the probability space $\left\{\Omega, \mathscr{F}_{t},\left\{\mathscr{F}_{t}\right\}_{t \geq 0}, \mathbb{P}\right\}$ with covariance operator $Q$. We assume that $-A$ and the covariance operator $Q$ of the Wiener process have the same eigenbasis $\left\{e_{m}\right\}_{m \geq 1}$ of $H$; that is,

$$
\begin{gathered}
-A e_{m}=\lambda_{m} e_{m}, \\
Q e_{m}=\alpha_{m} e_{m}, \quad m=1,2,3, \ldots,
\end{gathered}
$$

where $\left\{\lambda_{m}, m \in \mathbb{N}\right\}$ are the discrete spectrum of $-A$ and $0 \leq \lambda_{1} \leq \lambda_{2} \cdots \leq \lim _{m \rightarrow \infty} \lambda_{m}=\infty,\left\{\alpha_{m}, m \in \mathbb{N}\right\}$ are the eigenvalues of $Q$. Then, $W(t)$ is defined by

$$
W(t)=\sum_{n=1}^{\infty} \sqrt{\alpha_{m}} \beta_{m}(t) e_{m}, \quad t \geq 0,
$$

where $\beta_{m}(t)(m=1,2,3, \ldots)$ is a sequence of real-valued standard Brownian motions mutually independent of the probability space $\left.\left(\Omega, \mathscr{F}_{,}, \mathscr{F}_{t}\right\}_{t>0}, \mathbb{P}\right)$.

According to Da Prato and Zabczyk [1], we define stochastic integrals with respect to the Q-Wiener process $W(t)$. Let $K_{0}=Q^{1 / 2}(K)$ be the subspace of $K$ with the inner product $\langle u, v\rangle_{K_{0}}=\left\langle Q^{-1 / 2} u, Q^{-1 / 2} v\right\rangle_{K}$. Obviously, $K_{0}$ is a Hilbert space. Denote by $\mathscr{L}_{2}^{0}=\mathscr{L}\left(K_{0}, H\right)$ the family of Hilbert-Schmidt operators from $K_{0}$ into $H$ with the norm $\|\Psi\|_{\mathscr{L}_{2}^{0}}^{2}=\operatorname{tr}\left(\left(\Psi Q^{1 / 2}\right)\left(\Psi Q^{1 / 2}\right)^{*}\right)$.

Let $\Phi:(0, \infty) \rightarrow \mathscr{L}_{2}^{0}$ be a predictable, $\mathscr{F}_{t}$-adapted process such that

$$
\int_{0}^{t} \mathbb{E}\|\Phi(s)\|_{\mathscr{L}_{2}^{0}} d s \leq \infty, \quad \forall t>0 .
$$

Then, the $H$-valued stochastic integral $\int_{0}^{t} \Phi(s) d W(s)$ is a continuous square martingale. Let $N(d t, d u)$ be the Poisson measure which is independent of the $Q$-Wiener process $W(t)$. Denote the compensated or centered Poisson measure as

$$
\widetilde{N}(d t, d u)=N(d t, d u)-\rho d t \pi(d u),
$$

where $0<\rho<\infty$ is known as the jump rate and $\pi(\cdot)$ is the jump distribution (a probability measure). Let $\mathbb{Z} \in \mathscr{B}(K-\{0\})$ be the measurable set. Denote by $\mathscr{P}^{2}([0, T] \times \mathbb{Z}, H)$ the space of all predictable mappings $h:[0, T] \times \mathbb{Z} \rightarrow H$ for which

$$
\int_{0}^{T} \int_{\mathbb{Z}} \mathbb{E}\|h(t, u)\|_{H}^{2} d t \pi(d u)<\infty .
$$

Then, the $H$-valued stochastic integral

$$
\int_{0}^{T} \int_{\mathbb{Z}} h(t, u) \widetilde{N}(d t, d u)
$$

is a centred square-integrable martingale.

We recall the definition of the mild solution to (1) as follows.

Definition 1. A stochastic process $\{X(t): t \in[0, T]\}$ is called a mild solution of (1) if

(i) $X(t)$ is adapted to $\mathscr{F}_{t}, t \geq 0$, and has càdlàg path on $t \geq 0$ almost surely,

(ii) for arbitrary $t \in[0, T], \mathbb{P}\left\{w: \int_{0}^{t}\|X(s)\|_{H}^{2} d s<\infty\right\}=$ 1 , and almost surely

$$
\begin{aligned}
X(t)= & e^{t A} \xi(0)+\int_{0}^{t} e^{(t-s) A} f(X(s), X(s-\tau)) d s \\
& +\int_{0}^{t} e^{(t-s) A} g(X(s), X(s-\tau)) d W(s) \\
& +\int_{0}^{t} \int_{\mathbb{Z}} e^{(t-s) A} h(X(s), X(s-\tau), u) N(d s, d u)
\end{aligned}
$$

for any $X(t)=\xi(t) \in D_{\mathscr{F}_{0}}^{b}([-\tau, 0], H),-\tau \leq t \leq 0$.

For the existence and uniqueness of the mild solution to (1) (see [11]), we always make the following assumptions.

(H1) $(A, D(A))$ is a self-adjoint operator on $H$ such that $-A$ has discrete spectrum $0 \leq \lambda_{1} \leq \lambda_{2} \leq \cdots \leq$ $\lim _{m \rightarrow \infty} \lambda_{m}=\infty$ with corresponding eigenbasis $\left\{e_{m}\right\}_{m \geq 1}$ of $H$. In this case $A$ generates a compact $C_{0^{-}}$ semigroup $e^{t A}, t \geq 0$, such that $\left\|e^{t A}\right\| \leq e^{-\alpha t}$.

(H2) The mappings $f: H \times H \rightarrow H, g: H \times$ $H \rightarrow \mathscr{L}(K, H)$, and $h: H \times H \times \mathbb{Z} \rightarrow H$ are Borel measurable and satisfy the following Lipschitz continuity condition for some constant $L_{1}>0$ and arbitrary $x, y, x_{1}, y_{1}, x_{2}, y_{2} \in H$ and $u \in \mathbb{Z}$ :

$$
\begin{gathered}
\left\|f\left(x_{1}, y_{1}\right)-f\left(x_{2}, y_{2}\right)\right\|_{H}^{2} \\
\quad \vee\left\|g\left(x_{1}, y_{1}-g\left(x_{2}, y_{2}\right)\right)\right\|_{\mathscr{L}_{0}^{2}}^{2} \\
\leq L_{1}\left(\left\|x_{1}-x_{2}\right\|_{H}^{2}+\left\|y_{1}-y_{2}\right\|_{H}^{2}\right), \\
\left\|h\left(x_{1}, y_{1}, u\right)-h\left(x_{2}, y_{2}, u\right)\right\|_{H}^{2} \\
\leq L_{1}\left(\left\|x_{1}-x_{2}\right\|_{H}^{2}+\left\|y_{1}-y_{2}\right\|_{H}^{2}\right) .
\end{gathered}
$$

This further implies the linear growth condition; that is,

$$
\|f(x, y)\|_{H}^{2}+\|g(x, y)\|_{\mathscr{L}_{2}^{0}}^{2} \leq L_{0}\left(1+\|x\|_{H}^{2}+\|y\|_{H}^{2}\right),
$$

where

$$
L_{0}:=2\left(L_{2} \vee\|f(0,0)\|_{H}^{2} \vee\|g(0,0)\|_{\mathscr{L}_{2}^{0}}^{2}\right) .
$$


(H3) There exists $L_{2}>0$ satisfying

$$
\|h(x, y, u)\|_{H}^{2} \leq L_{2}\left(\|x\|_{H}^{2}+\|y\|_{H}^{2}\right),
$$

for each $x, y \in H$ and $u \in \mathbb{Z}$.

(H4) For $\xi \in D_{\mathscr{F}_{0}}^{b}([-\tau, 0], H)$, there exists a constant $L_{3}>0$ such that

$$
\mathbb{E}\left(|\xi(s)-\xi(t)|^{2}\right) \leq L_{3}|t-s|^{2}, \quad t, s \in[-\tau, 0] .
$$

We now describe our Euler-Maruyama scheme for the approximation of (1). For any $n \geq 1$, let $\pi_{n}: H \rightarrow$ $H_{n}=\operatorname{span}\left\{e_{1}, e_{2}, \ldots, e_{n}\right\}$ be the orthogonal projection; that is, $\pi_{n} x=\sum_{i=1}^{n}\left\langle x, e_{i}\right\rangle_{H} e_{i}, x \in H, A_{n}=\pi_{n} A, f_{n}=\pi_{n} f, g_{n}=\pi_{n} g$, and $h_{n}=\pi_{n} h$.

Consider the following stochastic differential delay equations with jumps on $H_{n}$ :

$$
\begin{aligned}
d X^{n}(t)= & {\left[A_{n} X^{n}(t)+f_{n}\left(X^{n}(t), X^{n}(t-\tau)\right)\right] d t } \\
& +g_{n}\left(X^{n}(t), X^{n}(t-\tau)\right) d W(t) \\
& +\int_{\mathbb{Z}} h_{n}\left(X^{n}(t), X^{n}(t-\tau), u\right) N(d t, d u), \\
& X^{n}(\theta)=\pi_{n} \xi(\theta), \quad \theta \in[-\tau, 0] .
\end{aligned}
$$

This spatial approximation (14) is called the Galerkin approximation of (1). Due to the fact that $\pi_{n} A x=$ $\pi_{n} A\left(\sum_{i=1}^{n}\left\langle x, e_{i}\right\rangle_{H} e_{i}\right)=-\sum_{i=1}^{n} \lambda_{i}\left\langle x, e_{i}\right\rangle_{H} e_{i}, x \in H_{n}$, it follows that for $x \in H_{n}, A_{n} x=A x, e^{t A_{n} x}=e^{t A x}$.

By (H2) and (H3) and the property of the projection operator, we have that

$$
\begin{gathered}
\left\|A_{n} x-A_{n} y\right\|_{H}^{2}=\left\|A_{n}(x-y)\right\|_{H}^{2} \leq \lambda_{n}^{2}\|x-y\|_{H}, \\
\left\|f_{n}\left(x_{1}, y_{1}\right)-f_{n}\left(x_{2}, y_{2}\right)\right\|_{H}^{2} \\
\vee\left\|g\left(x_{1}, y_{1}\right)-g\left(x_{2}, y_{2}\right)\right\|_{\mathscr{L}_{2}^{0}}^{2} \\
=\left\|f\left(x_{1}, y_{1}\right)-f\left(x_{2}, y_{2}\right)\right\|_{H}^{2} \\
\vee\left\|g\left(x_{1}, y_{1}\right)-g\left(x_{2}, y_{2}\right)\right\|_{\mathscr{L}_{2}^{0}}^{2} \\
\leq L_{1}\left(\left\|x_{1}-x_{2}\right\|_{H}^{2}+\left\|y_{1}-y_{2}\right\|_{H}^{2}\right), \\
\left\|h_{n}\left(x_{1}, y_{1}, u\right)-h_{n}\left(x_{2}, y_{2}, u\right)\right\|_{H}^{2} \\
=\left\|h\left(x_{1}, y_{1}, u\right)-h\left(x_{2}, y_{2}, u\right)\right\|_{H}^{2} \\
\leq L_{1}\left(\left\|x_{1}-x_{2}\right\|_{H}^{2}+\left\|y_{1}-y_{2}\right\|_{H}^{2}\right), \\
\left\|h_{n}(x, y, u)\right\|_{H}^{2}=\|h(x, y, u)\|_{H}^{2} \leq L_{2}\left(\|x\|_{H}^{2}+\|y\|_{H}^{2}\right)
\end{gathered}
$$

for arbitrary $x, y, x_{1}, x_{2}, y_{1}, y_{2} \in H_{n}$ and $u \in \mathbb{Z}$. Hence, (14) admits a unique solution $X^{n}(t)$ on $H_{n}$.

We introduce a time discretization scheme for (14) by using a stochastic exponential integrator. For given $T \geq 0$ and $\tau>0$, the time-step size $\Delta \in(0,1)$ is defined by $\Delta:=\tau / N$, for some sufficiently large integer $N>\tau$. For any integer $k \geq 0$, the time discretization scheme applied to (14) produces approximations $\bar{Y}^{n}(k \Delta) \approx X^{n}(k \Delta)$ by forming

$$
\begin{aligned}
& \bar{Y}^{n}((k+1) \Delta) \\
& =e^{\Delta A_{n}\{} \bar{Y}^{n}(k \Delta)+f_{n}\left(\bar{Y}^{n}(k \Delta), \bar{Y}^{n}(k \Delta-\tau)\right) \Delta \\
& \quad+g_{n}\left(\bar{Y}^{n}(k \Delta), \bar{Y}^{n}(k \Delta-\tau)\right) \Delta W_{k} \\
& \left.\quad+\int_{\mathbb{Z}} h_{n}\left(\bar{Y}^{n}(k \Delta), \bar{Y}^{n}(k \Delta-\tau), u\right) \Delta N_{k}(u)\right\}, \\
& \bar{Y}^{n}(\theta)=\pi_{n} \xi(\theta), \quad \theta \in[-\tau, 0],
\end{aligned}
$$

where $\Delta W_{k}=W((k+1) \Delta)-W(k \Delta)$ and $\Delta N_{k}(d u)=$ $N((0,(k+1) \Delta], d u)-N((0, k \Delta], d u)$.

The continuous-time version of this scheme associated with (14) is defined by

$$
\begin{aligned}
& Y^{n}(t) \\
& =e^{t A_{n}} Y^{n}(0)+\int_{0}^{t} e^{(t-\lfloor s\rfloor) A_{n}} f_{n}\left(Y^{n}(\lfloor s\rfloor), Y^{n}(\lfloor s\rfloor-\tau)\right) d s \\
& +\int_{0}^{t} e^{(t-\lfloor s\rfloor) A_{n}} g_{n}\left(Y^{n}(\lfloor s\rfloor), Y^{n}(\lfloor s\rfloor-\tau)\right) d W(s) \\
& +\int_{0}^{t} \int_{\mathbb{Z}} e^{(t-\lfloor s\rfloor) A_{n}} h_{n}\left(Y^{n}(\lfloor s\rfloor), Y^{n}(\lfloor s\rfloor-\tau), u\right) \\
& \quad \times N(d s, d u), \\
& Y^{n}(\theta)=\pi_{n} \xi(\theta), \quad \theta \in[-\tau, 0],
\end{aligned}
$$

where $\lfloor t\rfloor=[t / \Delta] \Delta$ with $[t / \Delta]$ denotes the integer of $t / \Delta$.

From (16) and (17), we have $Y^{n}(k \Delta)=\bar{Y}^{n}(k \Delta)$ for every $k \geq 0$. That is, the discrete-time and continuous-time schemes coincide at the grid points.

\section{Convergence Rate}

In this section, we shall investigate the convergence rate of the Euler-Maruyama method. In what follows, $C>0$ is a generic constant whose values may change from line to line.

Lemma 2. Let (H1)-(H4) hold; then there is a positive constant $C>0$ which depends on $T, \xi, L_{1}, L_{2}$, and $L_{3}$ but is independent of $\Delta$, such that

$$
\sup _{0 \leq t \leq T}\left(\mathbb{E}\|X(t)\|_{H}^{2}\right)^{1 / 2} \vee \sup _{0 \leq t \leq T}\left(\mathbb{E}\left\|Y^{n}(t)\right\|_{H}^{2}\right)^{1 / 2} \leq C .
$$


Proof. Due to the fact that $\left(\mathbb{E}\|\cdot\|_{H}^{2}\right)^{1 / 2}$ is a norm, we have from (8) that

$$
\begin{aligned}
(\mathbb{E} \| & \left.X(t) \|_{H}^{2}\right)^{1 / 2} \\
\leq & \left(\mathbb{E}\left\|e^{t A_{n}} \xi(0)\right\|_{H}^{2}\right)^{1 / 2} \\
& +\left(\mathbb{E}\left\|\int_{0}^{t} e^{(t-s) A} f(X(s), X(s-\tau)) d s\right\|_{H}^{2}\right)^{1 / 2} \\
& +\left(\mathbb{E}\left\|\int_{0}^{t} e^{(t-s) A} g(X(s), X(s-\tau)) d W(s)\right\|_{H}^{2}\right)^{1 / 2} \\
& +\left(\mathbb{E}\left\|\int_{0}^{t} e^{(t-s) A} h(X(s), X(s-\tau), u) N(d s, d u)\right\|_{H}^{2}\right)^{1 / 2} \\
= & \sum_{i=1}^{4} I_{i}(t) .
\end{aligned}
$$

Recall the property of the operator $A$ (see [18]):

$$
\begin{gathered}
\left\|(-A)^{\delta_{1}} e^{A t}\right\| \leq C t^{-\delta_{1}}, \\
\left\|(-A)^{\delta_{2}}\left(1-e^{A t}\right)\right\| \leq C t^{\delta_{2}}, \quad \delta_{1} \geq 0, \delta_{2} \in[0,1], \\
(-A)^{\alpha+\beta} x=(-A)^{\alpha}(-A)^{\beta} x, \quad x \in D\left((-A)^{r}\right),
\end{gathered}
$$

for $\alpha, \beta \in \mathbb{R}$, where $r=\max \{\alpha, \beta, \alpha+\beta\}$.

By $(\mathrm{H} 1)$ and (H2), together with the Minkowski integral inequality, we derive that

$$
\begin{gathered}
I_{2}(t) \leq \int_{0}^{t}\left(\mathbb{E}\left\|e^{(t-s) A} f(X(s), X(s-\tau))\right\|_{H}^{2}\right)^{1 / 2} d s \\
\leq C \int_{0}^{t}\left\{1+\left(\mathbb{E}\|X(s)\|_{H}^{2}\right)^{1 / 2}\right. \\
\left.+\left(\mathbb{E}\|X(s-\tau)\|_{H}^{2}\right)^{1 / 2}\right\} d s \\
\leq C+C \int_{0}^{t}\left(\mathbb{E}\|X(s)\|_{H}^{2}\right)^{1 / 2} d s .
\end{gathered}
$$

By (H1), (H2), and (H3) and using the Itô isometry, we have

$$
\begin{aligned}
& I_{3}(t)+I_{4}(t) \\
& \leq\left(\int_{0}^{t} \mathbb{E}\left\|e^{(t-s) A} g(X(s), X(s-\tau))\right\|_{\mathscr{L}_{2}^{0}}^{2} d s\right)^{1 / 2} \\
& +\left(\mathbb{E} \| \int_{0}^{t} \int_{Z} e^{(t-s) A} h(X(s), X(s-\tau), u) \widetilde{N}(d s, d u)\right. \\
& +\rho \int_{0}^{t} \int_{\mathbb{Z}} e^{(t-s) A} h \\
& \left.\quad \times(X(s), X(s-\tau), u) \pi(d u) \|_{H}^{2}\right)^{1 / 2}
\end{aligned}
$$

$$
\begin{aligned}
& \leq\left(\int_{0}^{t} L_{0}\left(1+\mathbb{E}\|X(s)\|_{H}^{2}+\mathbb{E}\|X(s-\tau)\|_{H}^{2}\right) d s\right)^{1 / 2} \\
& +\left(\mathbb{E} \| \int_{0}^{t} \int_{Z} e^{(t-s) A} h\right. \\
& \left.\quad \times(X(s), X(s-\tau), u) \widetilde{N}(d s, d u) \|_{H}^{2}\right)^{1 / 2} \\
& +\rho\left(\mathbb{E} \| \int_{0}^{t} \int_{\mathbb{Z}} e^{(t-s) A} h\right. \\
& \left.\quad \times(X(s), X(s-\tau), u) \pi(d u) d s \|_{H}^{2}\right)^{1 / 2} .
\end{aligned}
$$

Using Hölder inequality and (H3), for the last term of (22), we have

$$
\begin{gathered}
\rho\left(\mathbb{E}\left\|\int_{0}^{t} \int_{Z} e^{(t-s) A} h(X(s), X(s-\tau), u) \pi(d u) d s\right\|_{H}^{2}\right)^{1 / 2} \\
\leq C\left(\mathbb{E} \int_{0}^{t} \int_{Z}\|h(X(s), X(s-\tau), u)\|_{H}^{2} \pi(d u) d s\right)^{1 / 2} \\
\leq C \sqrt{L_{2}}\left(\int_{0}^{t}\left(\mathbb{E}\|X(s)\|_{H}^{2}+\mathbb{E}\|X(s-\tau)\|_{H}^{2}\right) d s\right)^{1 / 2} \\
\leq C \sqrt{L_{2}} \sqrt{\tau} \mathbb{E}\|\xi\|_{H}+p C \sqrt{2 L_{2}}\left(\int_{0}^{t} \mathbb{E}\|X(s)\|_{H}^{2} d s\right)^{1 / 2} .
\end{gathered}
$$

Moreover, by using the Itô isometry and (H3), we obtain that

$$
\begin{gathered}
\left(\mathbb{E}\left\|\int_{0}^{t} \int_{\mathbb{Z}} e^{(t-s) A} h(X(s), X(s-\tau), u) \widetilde{N}(d s, d u)\right\|_{H}^{2}\right)^{1 / 2} \\
\leq\left(\int_{0}^{t} \int_{\mathbb{Z}} \mathbb{E}\|h(X(s), X(s-\tau), u)\|_{H}^{2} \pi(d u) d s\right)^{1 / 2} \\
\leq \sqrt{L_{2}}\left(\int_{0}^{t}\left(\mathbb{E}\|X(s)\|_{H}^{2}+\mathbb{E}\|X(s-\tau)\|_{H}^{2}\right) d s\right)^{1 / 2} \\
\leq \sqrt{L_{2}} \sqrt{\tau} \mathbb{E}\|\xi\|_{H}+\sqrt{2 L_{2}}\left(\int_{0}^{t}\left(\mathbb{E}\|X(s)\|_{H}^{2} d s\right)^{1 / 2} .\right.
\end{gathered}
$$

Substituting (23) and (24) into (22), it follows that

$$
I_{3}(t)+I_{4}(t) \leq C+C \mathbb{E}\|\xi\|_{H}+C\left(\int_{0}^{t} \mathbb{E}\|X(s)\|_{H}^{2} d s\right)^{1 / 2} .
$$

Hence,

$$
\left(\mathbb{E}\|X(t)\|_{H}^{2}\right)^{1 / 2} \leq C+C \mathbb{E}\|\xi\|_{H}+C\left(\int_{0}^{t} \mathbb{E}\|X(s)\|_{H}^{2} d s\right)^{1 / 2} .
$$


Applying the Gronwall inequality, we have

$$
\sup _{0 \leq t \leq T}\left(\mathbb{E}\|X(t)\|_{H}^{2}\right)^{1 / 2} \leq C .
$$

Using the similar argument, the second assertion of (18) follows.

Lemma 3. Let (H1)-(H4) hold; for sufficiently small $\Delta$,

$$
\sup _{0 \leq t \leq T}\left(\mathbb{E}\|X(t)-X(\lfloor t\rfloor)\|_{H}^{2}\right)^{1 / 2} \leq C \Delta^{1 / 2},
$$

where $C>0$ is constant dependent on $T, \xi, L_{1}, L_{2}, L_{3}$, and $L_{4}$, while being independent of $\Delta$.

Proof. For any $t \in[0, T]$, we have from (8) that

$$
\begin{aligned}
X( & t)-X(\lfloor t\rfloor) \\
= & e^{\lfloor t\rfloor A}\left(e^{(t-\lfloor t\rfloor) A}-\mathbf{1}\right) \xi(0) \\
& +\int_{0}^{\lfloor t\rfloor}\left(e^{(t-\lfloor t\rfloor) A}-\mathbf{1}\right) e^{(\lfloor t\rfloor-s) A} f(X(s), X(s-\tau)) d s \\
& +\int_{\lfloor t\rfloor}^{t} e^{(t-s) A} f(X(s), X(s-\tau)) d s \\
& +\int_{0}^{\lfloor t\rfloor}\left(e^{(t-\lfloor t\rfloor) A}-\mathbf{1}\right) e^{(\lfloor t\rfloor-s) A} g(X(s), X(s-\tau)) d W(s) \\
& +\int_{0}^{\lfloor t\rfloor} \int_{\mathbb{Z}}\left(e^{(t-\lfloor t\rfloor) A}-\mathbf{1}\right) e^{(\lfloor t\rfloor-s) A} \\
& +\int_{\lfloor t\rfloor}^{t} e^{(t-s) A} g(X(s), X(s-\tau)) d W(s) \\
& +\int_{\lfloor t\rfloor}^{t} \int_{\mathbb{Z}} e^{(t-s) A} h(X(s), X(s-\tau), u) N(d s, d u) \\
= & \sum_{i=1}^{7} J_{i}(t) .
\end{aligned}
$$

Since $\left(\mathbb{E}\|\cdot\|_{H}^{2}\right)^{1 / 2}$ is a norm, it follows that

$$
\left(\mathbb{E}\|X(t)-X(\lfloor t\rfloor)\|_{H}^{2}\right)^{1 / 2} \leq \sum_{i=1}^{7}\left(\mathbb{E}\left\|J_{i}(t)\right\|_{H}^{2}\right)^{1 / 2} .
$$

Recalling the fundamental inequality $1-e^{-y} \leq y, y>0$, we get from (H1) that

$$
\begin{aligned}
& \left\|\left(e^{(t-\lfloor t\rfloor) A}-\mathbf{1}\right) x\right\|_{H}^{2} \\
& \quad=\left\|\sum_{i=1}^{\infty}\left(e^{-\lambda_{i}(t-\lfloor t\rfloor)}-1\right)\left\langle x, e_{i}\right\rangle e_{i}\right\|_{H}^{2} \\
& \leq\left(1-e^{-\lambda_{1}(t-\lfloor t\rfloor)}\right)^{2}\|x\|_{H}^{2} \\
& \leq \lambda_{1}^{2} \Delta^{2}\|x\|_{H}^{2} .
\end{aligned}
$$

Therefore,

$$
\begin{aligned}
\left(\mathbb{E}\left\|J_{1}(t)\right\|_{H}^{2}\right)^{1 / 2} & \\
\quad & \left(\mathbb{E}\left\|e^{\lfloor t\rfloor A}\left\{e^{(t-\lfloor t\rfloor) A}-\mathbf{1}\right\} \xi(0)\right\|_{H}^{2}\right)^{1 / 2} \\
& \leq \lambda_{1}\left(\mathbb{E}\|\xi(0)\|_{H}^{2}\right)^{1 / 2} \Delta .
\end{aligned}
$$

By (H1), (H2), and the Minkowski integral inequality, we obtain that

$$
\begin{aligned}
& \sum_{i=2}^{3}\left(\mathbb{E}\left\|J_{i}(t)\right\|_{H}^{2}\right)^{1 / 2} \\
& \leq \int_{0}^{\lfloor t\rfloor}\left\|e^{(t-\lfloor t\rfloor) A}-\mathbf{1}\right\|\left\|e^{(\lfloor t\rfloor-s) A}\right\| \\
& \quad \times\left(\mathbb{E}\|f(X(s), X(s-\tau))\|_{H}^{2}\right)^{1 / 2} d s \\
& +\int_{\lfloor t\rfloor}^{t}\left(\mathbb{E}\|f(X(s), X(s-\tau))\|_{H}^{2}\right)^{1 / 2} d s .
\end{aligned}
$$

Together with (31), we arrive at

$$
\begin{aligned}
& \sum_{i=2}^{3}\left(\mathbb{E}\left\|J_{i}(t)\right\|_{H}^{2}\right)^{1 / 2} \\
& \quad \leq\left(\lambda_{1} \Delta \int_{0}^{\lfloor t\rfloor} d s+\Delta\right) C \sup _{0 \leq t \leq T}\left(\mathbb{E}\|f(X(t), X(t-\tau))\|_{H}^{2}\right)^{1 / 2} \\
& \quad \leq C\left(1+\sup _{0 \leq t \leq T}\left(\mathbb{E}\|X(t)\|_{H}^{2}\right)^{1 / 2}\right) \Delta .
\end{aligned}
$$

Following the argument of (22), we derive that

$$
\begin{aligned}
& \sum_{i=4}^{7}\left(\mathbb{E}\left\|J_{i}(t)\right\|_{H}^{2}\right)^{1 / 2} \\
& \leq\left(\int_{0}^{\lfloor t\rfloor}\left\|e^{(t-\lfloor t\rfloor) A}-1\right\|^{2}\left\|e^{(\lfloor t\rfloor-s) A}\right\|^{2}\right. \\
& \left.\times \mathbb{E}\|g(X(s), X(s-\tau))\|_{\mathscr{L}_{2}^{0}}^{2} d s\right)^{1 / 2} \\
& +C\left(\int_{0}^{\lfloor t\rfloor} \int_{\mathbb{Z}}\left\|e^{(t-\lfloor t\rfloor) A}-1\right\|^{2}\left\|e^{(\lfloor t\rfloor-s) A}\right\|^{2}\right. \\
& \left.\times \mathbb{E}\|h(X(s), X(s-\tau), u)\|_{H}^{2} \pi(d u) d s\right)^{1 / 2} \\
& +\left(\int_{\lfloor t\rfloor}^{t}\left\|e^{(t-s) A}\right\|^{2} \mathbb{E}\|g(X(s), X(s-\tau))\|_{\mathscr{L}_{2}^{0}}^{2} d s\right)^{1 / 2} \\
& +C\left(\int_{\lfloor t\rfloor}^{t} \int_{\mathbb{Z}}\left\|e^{(t-s) A}\right\|^{2}\right. \\
& \left.\times \mathbb{E}\|h(X(s), X(s-\tau), u)\|_{H}^{2} \pi(d u) d s\right)^{1 / 2} \\
& \leq C\left(1+\sup _{0 \leq t \leq T}\left(\mathbb{E}\|X(t)\|_{H}^{2}\right)^{1 / 2}\right) \Delta^{1 / 2} .
\end{aligned}
$$


Substituting (32), (34), and (35) into (30), we arrive at

$$
\begin{aligned}
& \left(\mathbb{E}\|X(t)-X(\lfloor t])\|_{H}^{2}\right)^{1 / 2} \\
& \quad \leq C\left(1+\sup _{0 \leq t \leq T}\left(\mathbb{E}\|X(t)\|_{H}^{2}\right)^{1 / 2}\right) \Delta^{1 / 2} .
\end{aligned}
$$

Therefore, by Lemma 2, the required assertion (28) follows.

Now, we state our main result in this paper as follows.

Theorem 4. Let (H1)-(H4) hold, and

$$
\sqrt{L_{1}}\left(2 \alpha^{-1}+(\rho+3)(2 \alpha)^{-1 / 2}\right)<1
$$

Then,

$$
\sup _{0 \leq t \leq T}\left(\mathbb{E}\left\|X(t)-Y^{n}(t)\right\|_{H}^{2}\right)^{1 / 2} \leq C\left\{\lambda_{n}^{-1 / 2}+\Delta^{1 / 2}\right\}
$$

where $C>0$ is a constant dependent on $T, \xi, L_{1}, L_{2}, L_{3}$, and $L_{4}$, while being independent of $n$ and $\Delta$.

Proof. By (8) and (17), we obtain

$$
\begin{aligned}
& X(t)-Y^{n}(t) \\
& =e^{t A}\left(1-\pi_{n}\right) \xi(0) \\
& +\int_{0}^{t} e^{(t-s) A}(f(X(s), X(s-\tau)) \\
& \left.-f_{n}(X(s), X(s-\tau))\right) d s \\
& +\int_{0}^{t} e^{(t-s) A}\left(f_{n}(X(s), X(s-\tau))\right. \\
& \left.-f_{n}(X(\lfloor s\rfloor), X(\lfloor s\rfloor-\tau))\right) d s \\
& +\int_{0}^{t} e^{(t-s) A}\left(g_{n}(X(s), X(s-\tau))\right. \\
& \left.-g_{n}(X(\lfloor s\rfloor), X(\lfloor s\rfloor-\tau))\right) d W(s) \\
& +\int_{0}^{t} e^{(t-s) A}\left(f_{n}(X(\lfloor s\rfloor), X(\lfloor s\rfloor-\tau))\right. \\
& \left.-f_{n}\left(Y^{n}(\lfloor s\rfloor), Y^{n}(\lfloor s\rfloor-\tau)\right)\right) d s \\
& +\int_{0}^{t} e^{(t-s) A}\left(g_{n}(X(\lfloor s\rfloor), X(\lfloor s\rfloor-\tau))\right. \\
& \left.-g_{n}\left(Y^{n}(\lfloor s\rfloor), Y^{n}(\lfloor s\rfloor-\tau)\right)\right) d W(s)
\end{aligned}
$$

$$
\begin{aligned}
& +\int_{0}^{t} e^{(t-s) A}(g(X(s), X(s-\tau)) \\
& \left.\quad-g_{n}(X(s), X(s-\tau))\right) d W(s) \\
& +\int_{0}^{t} e^{(t-s) A}\left(1-e^{(s-\lfloor s\rfloor) A}\right) f_{n}\left(Y^{n}(\lfloor s\rfloor), Y^{n}(\lfloor s\rfloor-\tau)\right) d s \\
& +\int_{0}^{t} e^{(t-s) A}\left(1-e^{(s-\lfloor s\rfloor) A}\right) \\
& \quad \times g_{n}\left(Y^{n}(\lfloor s\rfloor), Y^{n}(\lfloor s\rfloor-\tau)\right) d W(s)
\end{aligned}
$$$$
+\int_{0}^{t} \int_{\mathbb{Z}} e^{(t-s) A}\{h(X(s), X(s-\tau), u)
$$$$
\left.-h_{n}(X(s), X(s-\tau), u)\right\} N(d s, d u)
$$$$
+\int_{0}^{t} \int_{\mathbb{Z}} e^{(t-s) A}\left\{h_{n}(X(s), X(s-\tau), u)\right.
$$$$
\left.-h_{n}(X(\lfloor s\rfloor), X(\lfloor s\rfloor-\tau), u)\right\} N(d s, d u)
$$$$
+\int_{0}^{t} \int_{\mathbb{Z}} e^{(t-s) A}\left\{h_{n}(X(\lfloor s\rfloor), X(\lfloor s\rfloor-\tau), u)-h_{n}\right.
$$$$
\left.\times\left(Y^{n}(\lfloor s\rfloor), Y^{n}(\lfloor s\rfloor-\tau), u\right)\right\} N(d s, d u)
$$$$
+\int_{0}^{t} \int_{Z} e^{(t-s) A}\left(1-e^{(s-\lfloor s\rfloor) A}\right) h_{n}
$$$$
\times\left(Y^{n}(\lfloor s\rfloor), Y^{n}(\lfloor s\rfloor-\tau), u\right) N(d s, d u)
$$$$
=\sum_{i=1}^{13} K_{i}(t) \text {. }
$$

Noting that $\left(\mathbb{E}\|\cdot\|_{H}^{2}\right)^{1 / 2}$ is a norm, we have

$$
\left(\mathbb{E}\left\|X(t)-Y^{n}(t)\right\|_{H}^{2}\right)^{1 / 2} \leq \sum_{i=1}^{13}\left(\mathbb{E}\left\|K_{i}(t)\right\|_{H}^{2}\right)^{1 / 2}
$$

By (H1) and the nondecreasing spectrum $\left\{\lambda_{m}\right\}_{m \geq 1}$, it easily follows that

$$
\begin{aligned}
\mathbb{E} \| e^{t A} & \left(1-\pi_{n}\right) \xi(0) \|_{H} \\
& =\mathbb{E}\left(\sum_{m=n+1}^{\infty} e^{-2 \lambda_{m} t}\left\langle\xi(0), e_{m}\right\rangle_{H}^{2}\right)^{1 / 2} \\
& =\mathbb{E}\left(\sum_{m=n+1}^{\infty} \frac{e^{-2 \lambda_{m} t}}{\lambda_{m}^{2}} \lambda_{m}^{2}\left\langle\xi(0), e_{m}\right\rangle_{H}^{2}\right)^{1 / 2} \\
& \leq \frac{1}{\lambda_{n}} \mathbb{E}\|A \xi(0)\|_{H} .
\end{aligned}
$$


By (H2), the Minkowski integral inequality, and Lemma 2, we have

$$
\begin{aligned}
&(\mathbb{E}\left.\left\|K_{2}(t)\right\|_{H}^{2}\right)^{1 / 2} \\
& \leq \int_{0}^{t}\left(\mathbb{E}\left\|e^{(t-s) A}\left(1-\pi_{n}\right) f(X(s), X(s-\tau))\right\|_{H}^{2}\right)^{1 / 2} d s \\
&=\int_{0}^{t}\left(\mathbb{E} \sum_{m=n+1}^{\infty} e^{-2 \lambda_{m}(t-s)}\left\langle f(X(s), X(s-\tau)), e_{m}\right\rangle_{H}^{2}\right)^{1 / 2} d s \\
& \leq \int_{0}^{t} e^{-\lambda_{n}(t-s)}\left(\mathbb{E} \sum_{m=n+1}^{\infty}\left\langle f(X(s), X(s-\tau)), e_{m}\right\rangle_{H}^{2}\right)^{1 / 2} d s \\
& \leq C \int_{0}^{t} e^{-\lambda_{n}(t-s)} \\
& \quad \times\left\{1+\left(\mathbb{E}\|X(s)\|_{H}^{2}\right)^{1 / 2}+\left(\mathbb{E}\|X(s-\tau)\|_{H}^{2}\right)^{1 / 2}\right\} d s \\
& \leq C \lambda_{n}^{-1} .
\end{aligned}
$$

Applying (H1), (H2), and Lemma 3 and combining the Minkowski integral inequality and the Itô isometry yield

$$
\begin{aligned}
\sum_{i=3}^{6}(\mathbb{E} & \left.\left\|K_{i}(t)\right\|_{H}^{2}\right)^{1 / 2} \\
\leq & \sqrt{L_{1}} \int_{0}^{t}\left\|e^{(t-s) A}\right\|\left(\mathbb { E } \left(\|X(s)-X(\lfloor s\rfloor)\|_{H}^{2}\right.\right. \\
& \left.\left.+\|X(s-\tau)-X(\lfloor s\rfloor-\tau)\|_{H}^{2}\right)\right)^{1 / 2} d s \\
& +\sqrt{L_{1}} \int_{0}^{t}\left\|e^{(t-s) A}\right\|\left(\mathbb { E } \left(\left\|X(\lfloor s\rfloor)-Y^{n}(\lfloor s\rfloor)\right\|_{H}^{2}\right.\right. \\
& \left.\left.+\left\|X(\lfloor s\rfloor-\tau)-Y^{n}(\lfloor s\rfloor-\tau)\right\|_{H}^{2}\right)\right)^{1 / 2} d s \\
& +\sqrt{L_{1}}\left(\int _ { 0 } ^ { t } \| e ^ { ( t - s ) A } \| ^ { 2 } \left(\mathbb { E } \left(\|X(s)-X(\lfloor s\rfloor)\|_{H}^{2}\right.\right.\right. \\
& +\sqrt{L_{1}}\left(\int _ { 0 } ^ { t } \| e ^ { ( t - s ) A } \| ^ { 2 } \left(\mathbb{E}\left\|X(\lfloor s\rfloor)-Y^{n}(\lfloor s\rfloor)\right\|_{H}^{2}\right.\right. \\
& +\sqrt{L_{1}} \sup _{0 \leq s \leq t}\left(\mathbb{E}\left\|X(s)-Y^{n}(s)\right\|_{H}^{2}\right)^{1 / 2}\left(\int_{-\tau}^{t / 2}+\sqrt{L_{1}} \sup _{0 \leq s \leq t}\left(\mathbb{E}\left\|X(s)-Y^{n}(s)\right\|_{H}^{2}\right)^{1 / 2}(2 \alpha)^{-1 / 2}\right) . \\
& \left.\left.+\sqrt{L_{1}} \sup _{0 \leq s \leq t}\left(\mathbb{E}\left\|X(s)-Y^{n}(\lfloor s\rfloor-\tau)\right\|_{H}^{2}\right)\right) d s\right)^{1 / 2}
\end{aligned}
$$

By the Itô isometry and a similar argument to that of (42), we deduce that

$$
\begin{aligned}
& \left(\mathbb{E}\left\|K_{7}(t)\right\|_{H}^{2}\right)^{1 / 2} \\
& \quad \leq\left(\int_{0}^{t} \mathbb{E}\left\|e^{(t-s) A}\left(1-\pi_{n}\right) g(X(s), X(s-\tau))\right\|_{\mathscr{L}_{2}^{0}}^{2} d s\right)^{1 / 2} \\
& \quad \leq C\left(\int_{0}^{t} e^{-2 \lambda_{n}(t-s)} \mathbb{E}\|g(X(s), X(s-\tau))\|_{\mathscr{L}_{2}^{0}}^{2} d s\right)^{1 / 2} \\
& \quad \leq C \lambda_{n}^{-1 / 2} .
\end{aligned}
$$

Moreover, by (31), (H2), and Lemma 2 and combining the Minkowski integral inequality and the Itô isometry, we have

$$
\begin{aligned}
& \sum_{i=8}^{9}\left(\mathbb{E}\left\|K_{i}(t)\right\|_{H}^{2}\right)^{1 / 2} \\
& \leq \int_{0}^{t}\left(\mathbb{E}\left\|e^{(t-s) A}\left(1-e^{(s-\lfloor s\rfloor) A}\right)\right\|^{2}\right. \\
& \left.\quad \times\left\|f_{n}\left(Y^{n}(\lfloor s\rfloor), Y^{n}(\lfloor s\rfloor-\tau)\right)\right\|_{H}^{2}\right)^{1 / 2} d s \\
& +\left(\int_{0}^{t} \mathbb{E}\left\|e^{(t-s) A}\left(1-e^{(s-\lfloor s\rfloor) A}\right)\right\|^{2}\right. \\
& \left.\quad \times\left\|g_{n}\left(Y^{n}(\lfloor s\rfloor), Y^{n}(\lfloor s\rfloor-\tau)\right)\right\|_{H}^{2} d s\right)^{1 / 2} \\
& \leq C \Delta \int_{0}^{t}\left(\mathbb{E}\left\|f_{n}\left(Y^{n}(\lfloor s\rfloor), Y^{n}(\lfloor s\rfloor-\tau)\right)\right\|_{H}^{2}\right)^{1 / 2} d s \\
& +C \Delta\left(\int_{0}^{t} \mathbb{E}\left\|g_{n}\left(Y^{n}(\lfloor s\rfloor), Y^{n}(\lfloor s\rfloor-\tau)\right)\right\|_{\mathscr{L}_{2}^{0}}^{2} d s\right)^{1 / 2} \\
& \leq C \Delta .
\end{aligned}
$$

By (31) and the Itô isometry, we obtain that

$$
\begin{aligned}
& \left(\mathbb{E}\left\|K_{10}(t)\right\|_{H}^{2}\right)^{1 / 2} \\
& \leq\left(\mathbb{E} \| \int_{0}^{t} \int_{\mathbb{Z}} e^{(t-s) A}\left(1-\pi_{n}\right) h\right. \\
& \left.\times(X(s), X(s-\tau), u) \widetilde{N}(d s, d u) \|_{H}^{2}\right)^{1 / 2} \\
& +\rho\left(\mathbb{E} \| \int_{0}^{t} \int_{\mathbb{Z}} e^{(t-s) A}\left(1-\pi_{n}\right) h\right. \\
& \left.\leq\left(\int_{0}^{t} \int_{\mathbb{Z}}\left\|e^{(t-s) A}\left(1-\pi_{n}\right)\right\|^{2} \mathbb{E}(s), X(s-\tau), u\right) \pi(d u) d s \|_{H}^{2}\right)^{1 / 2} \\
& \left.\times\|h(X(s), X(s-\tau), u)\|_{H}^{2} \pi(d u) d s\right)^{1 / 2}
\end{aligned}
$$




$$
\begin{aligned}
& \quad+\rho\left(\int_{0}^{t} \int_{\mathbb{Z}}\left\|e^{(t-s) A}\left(1-\pi_{n}\right)\right\|^{2}\right. \\
& \left.\quad \times \mathbb{E}\|h(X(s), X(s-\tau), u)\|_{H}^{2} \pi(d u) d s\right)^{1 / 2} \\
& \leq C\left(\int_{0}^{t} e^{-2 \lambda_{n}(t-s)}\left(\mathbb{E}\|X(s)\|_{H}^{2}+\mathbb{E}\|X(s-\tau)\|_{H}^{2}\right) d s\right)^{1 / 2} \\
& \leq C \lambda_{n}^{-1 / 2} .
\end{aligned}
$$

Carrying out the similar arguments to those of (43) and (45), we derive that

$$
\begin{gathered}
\left(\mathbb{E}\left\|K_{11}(t)\right\|_{H}^{2}\right)^{1 / 2}+\left(\mathbb{E}\left\|K_{12}(t)\right\|_{H}^{2}\right)^{1 / 2} \\
\leq C \Delta^{1 / 2}+(2 \alpha)^{-1 / 2}(\rho+1) \\
\times \sqrt{L_{1}} \sup _{0 \leq s \leq t}\left(\mathbb{E}\left\|X(s)-Y^{n}(s)\right\|_{H}^{2}\right)^{1 / 2}, \\
\left(\mathbb{E}\left\|K_{13}(t)\right\|_{H}^{2}\right)^{1 / 2} \leq C \Delta .
\end{gathered}
$$

As a result, putting (41)-(47) into (40) gives that

$$
\begin{aligned}
\sup _{0 \leq s \leq t}(\mathbb{E} & \left.\left\|X(s)-Y^{n}(s)\right\|_{H}^{2}\right)^{1 / 2} \\
\leq & C \lambda_{n}^{-1 / 2}+C \Delta^{1 / 2}+\sqrt{L_{1}}\left(2 \alpha^{-1}+(\rho+3)(2 \alpha)^{-1 / 2}\right) \\
& \times \sup _{0 \leq s \leq t}\left(\mathbb{E}\left\|X(s)-Y^{n}(s)\right\|_{H}^{2}\right)^{1 / 2},
\end{aligned}
$$

and therefore the desired assertion follows.

Remark 5. For finite-dimensional Euler-Maruyama method, the condition (37) can be deleted by the Gronwall inequality $[16,17]$.

\section{Acknowledgment}

This work is partially supported by National Natural Science Foundation of China Under Grants 60904005 and 11271146.

\section{References}

[1] G. Da Prato and J. Zabczyk, Stochastic Equations in Infinite Dimensions, vol. 44 of Encyclopedia of Mathematics and Its Applications, Cambridge University Press, Cambridge, UK, 1992.

[2] K. Liu, Stability of Infinite Dimensional Stochastic Differential Equations with Applications, Chapman \& Hall/CRC, Boca Raton, Fla, USA, 2004.

[3] Q. Luo, F. Deng, J. Bao, B. Zhao, and Y. Fu, "Stabilization of stochastic Hopfield neural network with distributed parameters," Science in China F, vol. 47, no. 6, pp. 752-762, 2004.

[4] Q. Luo, F. Deng, X. Mao, J. Bao, and Y. Zhang, "Theory and application of stability for stochastic reaction diffusion systems," Science in China F, vol. 51, no. 2, pp. 158-170, 2008.
[5] X. Mao, Stochastic Differential Equations and Applications, Horwood Publishing, Chichester, UK, 2007.

[6] Y. Shen and J. Wang, "An improved algebraic criterion for global exponential stability of recurrent neural networks with timevarying delays," IEEE Transactions on Neural Networks, vol. 19, no. 3, pp. 528-531, 2008.

[7] Y. Shen and J. Wang, "Almost sure exponential stability of recurrent neural networks with markovian switching," IEEE Transactions on Neural Networks, vol. 20, no. 5, pp. 840-855, 2009.

[8] I. Gyöngy and N. Krylov, "Accelerated finite difference schemes for linear stochastic partial differential equations in the whole space," SIAM Journal on Mathematical Analysis, vol. 42, no. 5, pp. 2275-2296, 2010.

[9] A. Jentzen, P. E. Kloeden, and G. Winkel, "Efficient simulation of nonlinear parabolic SPDEs with additive noise," The Annals of Applied Probability, vol. 21, no. 3, pp. 908-950, 2011.

[10] P. E. Kloeden, G. J. Lord, A. Neuenkirch, and T. Shardlow, "The exponential integrator scheme for stochastic partial differential equations: pathwise error bounds," Journal of Computational and Applied Mathematics, vol. 235, no. 5, pp. 1245-1260, 2011.

[11] J. Bao, A. Truman, and C. Yuan, "Stability in distribution of mild solutions to stochastic partial differential delay equations with jumps," Proceedings of The Royal Society of London A, vol. 465, no. 2107, pp. 2111-2134, 2009.

[12] B. Boufoussi and S. Hajji, "Successive approximation of neutral functional stochastic differential equations with jumps," Statistics and Probability Letters, vol. 80, no. 5-6, pp. 324-332, 2010.

[13] E. Hausenblas, "Finite element approximation of stochastic partial differential equations driven by Poisson random measures of jump type," SIAM Journal on Numerical Analysis, vol. 46, no. 1, pp. 437-471, 2007/08.

[14] S. Peszat and J. Zabczyk, Stochastic Partial Differential Equations with Lévy Noise: An Evolution Equation Approach, vol. 113 of Encyclopedia of Mathematics and Its Applications, Cambridge University Press, Cambridge, UK, 2007.

[15] M. Röckner and T. Zhang, "Stochastic evolution equations of jump type: existence, uniqueness and large deviation principles," Potential Analysis, vol. 26, no. 3, pp. 255-279, 2007.

[16] J. Bao, B. Böttcher, X. Mao, and C. Yuan, "Convergence rate of numerical solutions to SFDEs with jumps," Journal of Computational and Applied Mathematics, vol. 236, no. 2, pp. 119-131, 2011.

[17] N. Jacob, Y. Wang, and C. Yuan, "Numerical solutions of stochastic differential delay equations with jumps," Stochastic Analysis and Applications, vol. 27, no. 4, pp. 825-853, 2009.

[18] A. Pazy, Semigroups of Linear Operators and Applications to Partial Differential Equations, vol. 44 of Applied Mathematical Sciences, Springer, New York, NY, USA, 1983. 


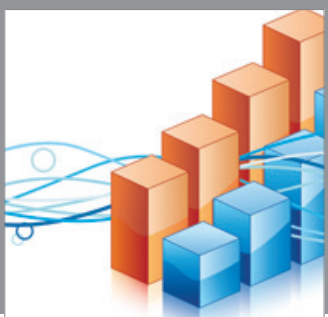

Advances in

Operations Research

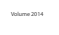

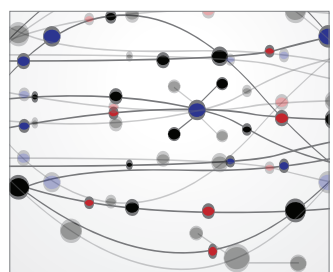

\section{The Scientific} World Journal
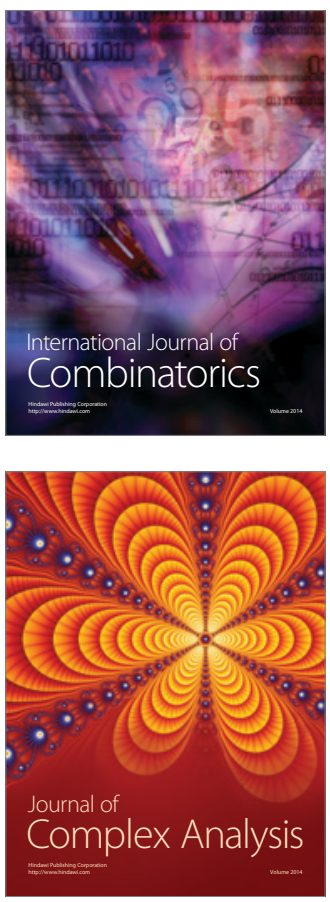

International Journal of

Mathematics and

Mathematical

Sciences
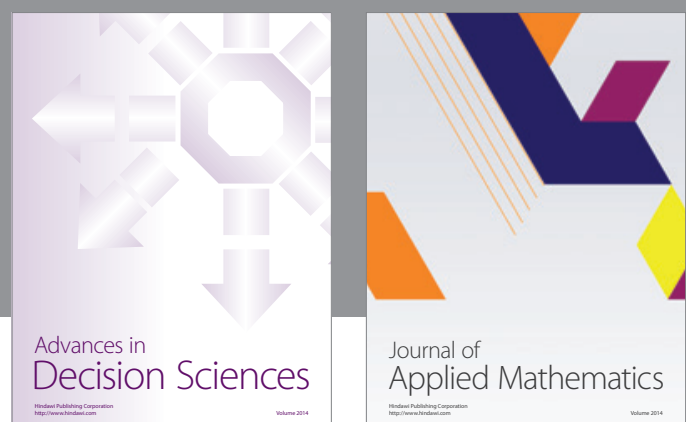

Journal of

Applied Mathematics
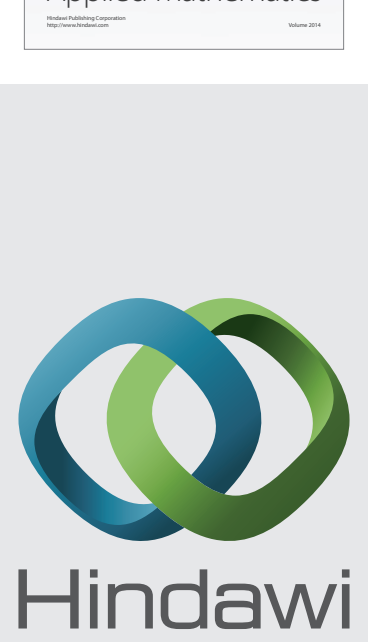

Submit your manuscripts at http://www.hindawi.com
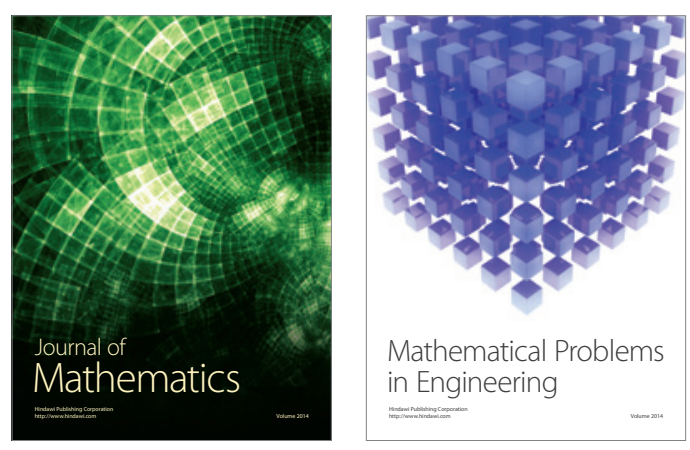

Mathematical Problems in Engineering
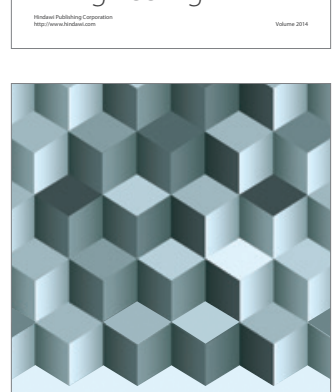

Journal of

Function Spaces
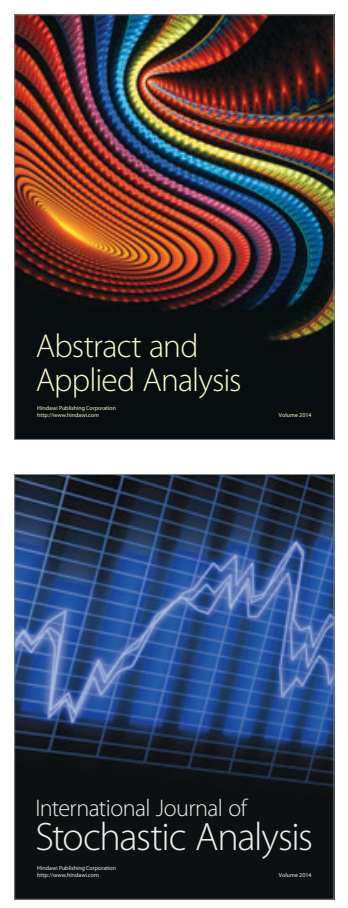

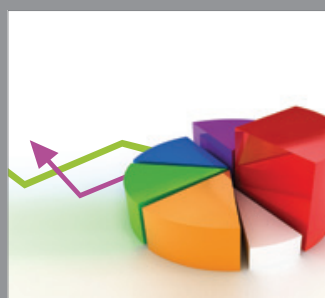

ournal of

Probability and Statistics

Promensencen
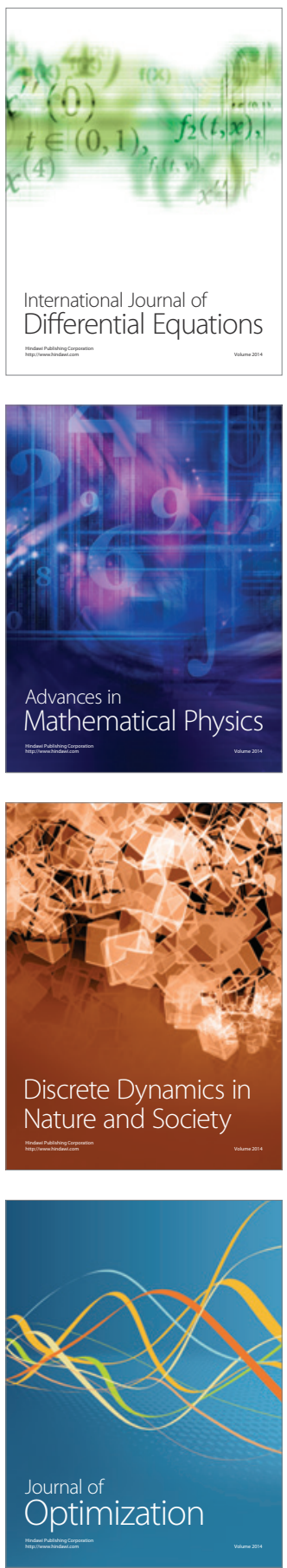